\title{
Revised
}

\section{Megasonic sonication for cost-effective and automatable elution of Cryptosporidiumfrom filters and membranes}

4 Abdelfateh Kerrouche ${ }^{1,2, *}$, Marc P.Y. Desmulliez ${ }^{1}$ and Helen Bridle ${ }^{2}$

5

6

7

1) Heriot-Watt University, MicroSystems Engineering Centre (MISEC), Riccarton, Edinburgh, United Kingdom, EH14 4AS

2) Heriot-Watt University, Institute of Biological Chemistry, Biophysics and Bioengineering (IB3), Riccarton, Edinburgh, United Kingdom, EH14 4AS

* Corresponding author: Email addresses: a.kerrouche@hw.ac.uk /

abdf.kerrouche@hotmail.co.uk

Tel.: +44 (0) 131650 5814; fax: +44 (0) 1316506554

\begin{abstract}
Sample processing is a highly challenging stage inthe monitoring of waterborne pathogens.

This step is time-consuming, requires highly trained technicians and often results in low recovery rates of pathogens. In the UK but also in other parts of the world, Cryptosporidiumis the only pathogen directly tested for in routine operational monitoring. The traditional sampling process involves the filtration of 1000L of water, semi-automated elution of the filters and membranes with recovery rates of about $30-40 \%$ typically. This paper explores the use of megasonic sonication in an attempt to increase recovery rates and reduce both the time required for processing and the number of labour-intensive steps. Results demonstrate that megasonic energy assisted elution is equally effective as the traditional manual process in terms of recovery rates. Major advantages are however offered in terms ofreduction of the elution volume enabling the current centrifugation stage to be avoided. This saves time, equipment and staff costs and critically removes the step in the process that would be most
\end{abstract}


challenging to automate, paving the way thereby for highly effective automated solutions to pathogens monitoring.

Keywords:Cryptosporidium; elution; megasonic agitation; sonication; filtration; waterborne pathogens; monitoring.

\section{Introduction}

The presence of pathogens in drinking water is amajor cause of disease outbreaks and endemic levels of illness, impacting upon productivity as well as quality of living(World Health Organisation,2011;Hrudeyet al., 2003). Water quality compromised by microbial contamination is also a concern for food producers and several disease outbreaks have been linked to the water utilised in food production(Söderströmet al.,2008; Brughaet al.,1999).Although the labour-intensive monitoring of the water supply for the presence of pathogens can be expensive, such measures allow the reduction of the costs associated with disease outbreaks.

Cryptosporidium is a particularly problematic pathogen in this regard.This protozoa has a low infectious dose, a longevity of months in the water environment and a high resistance to disinfection by chlorination. Despite the removal of the regulatory requirement to directly test for the presence ofCryptosporidium in water, UK water utilities continue to perform regular, even daily, checks at many sites.Because of their low infectious dose, sample preparation is required to concentrate waterborne pathogens from a large volume of water, of the order ofthousands of litres, to a small sample such as a few $\mu$ Ls to be used by detection devices (Bridle, 2013).Detection protocols such as the U.S. Environmental Protection Agency (EPA) method 1623.1(Method 1623.1, 2012) or the UK Environment Agency Blue Book 
publications (UK Environment Agency, 2010)stipulate a procedure forCryptosporidium detection. This method consists of several steps involving filtration (1000L/24hrs), elution stage 1 (remove oocysts from filter into $1200 \mathrm{~mL}$ ), elution stage 2 (concentrate the elutate using a membrane to $50 \mathrm{~mL}$ ), centrifugation (centrifugation to $5 \mathrm{~mL}$ ), enrichment (immunomagnetic separation IMS to separate oocysts from other particulate matter to $50 \mu \mathrm{L}$ ) and detection (staining with fluorescent dyes followed by microscopic examination for identification). Most of these stages require a long time, large and/or specialised equipment or highly qualified staff.

Elution steps are critical in ensuring a high recovery rate of pathogens(Francyet al., 2013). Manufacturers of commercially available filters report rates in excess of $70 \%$. However, personal communications with water utilities suggest that recovery rates do not often reach these levels. This is further confirmed by results of a variety of literature studies in which recovery rates on the order of $30 \%$ to $40 \%$ were repeatedly measured across a range of different water types(Polaczyk et al., 2008; Smith and Hill, 2009; Leskinenet al., 2010; Mull and Hill, 2009) or across a range of filters using lake water samples (Francy et al., 2013).

In this paper we explore the use of a novel physical approach to filter and membrane elution, namely the use of megasonic sonication as a replacement to manual processes of filter elution.In the last few years,megasonic wave assisted cleaning systems have been widely used to clean various types of objects possessing complex surface geometriessuch aselectronic devices,semiconductor wafers or component parts(Kaufmann et al., 2008; Busnaina et al., 1995; Helbig et al., 2008). In megasonic assisted agitation, apiezoelectric transducer, placed inside a tank, produces high frequency sound waves, typically over 1 $\mathrm{MHz}$, that propagate through the liquid. Each point along the sound wave oscillates between a maximum and a minimum pressure. When the minimum pressure is below the vapour 
pressure of the liquid, bubbles are formed. As the pressure increases to the maximum pressure, the bubblesimplodecreating local turbulence at the implosion sites (Chitra et al., 2004).Megasonic waves propagate at a higher frequencythan ultrasonic waves.Smaller bubbles with less resulting cavitation energy are created, resulting in a gentler elution and potentially avoiding destructionof the pathogens(Al-Sabi et al., 2011).

Studies on the effect of the sonication of filters using ultrasound were performed to elute bacteria from filters for safe drinking water (Mendez et al., 2004) or from food samples (Ruban et al., 2011).

\section{The effects of ultrasound with different sonication power and time durations on waterborne} protozoa Cryptosporidium and Giardia were studied. The results showed that changes in parasite characteristics became visible (the shells were broken) when sonication time was extended (Al-Sabi et al., 2011).A study investigated the effect of underwater ultrasound on the viability of Cryptosporidium oocysts and demonstrated that more than $90 \%$ of the dispersed Cryptosporidium oocysts could be deactivated in few minutes ofcontinuous sonication(Ashokkumar et al., 2003). However, the deactivation of oocysts by this method is undesirable if one wishes to preserve the viability of the pathogens for further determination of their infectivity. Additionally, DNA degradation could be incompatible with the molecular tools currently under development(Bridle et al., 2014). In contrast, through the minimisation of the time required for bubble growth, megasonic sonication offers a way to elute undamaged and potentially viable oocysts from filters and membranes. This paper presents, for the first time, the use of megasonic sonication for pathogen elution and evaluates its qualities in terms of recovery rates, pathogen viability, processing,time required and potential for automation. 


\section{Materials and methods}

\subsection{Standard elution protocol}

The standard elution procedure as recommended in the U.S. Environmental Protection Agency (EPA) method 1623.1 (Method 1623.1, 2012) or the UK Environment Agency Blue Book publications (UK Environment Agency, 2010) is used by the water utility company, Scottish Water, which assisted in the microscopic evaluation of oocysts following the different elution protocols.The Filta-Max sponge filter from the IDEXX company, is first removed from the filter housingand placed into a washing station which encompasses a concentrator unit. In this washing station the filter is rinsed twice with $600 \mathrm{~mL}$ ofPhosphateBuffered Saline with Tween ${ }^{\circledR} 20$ (PBST) for about 20 minutes although the duration of the rinsing time depends on the water sample. The wash solution is then passed through a membrane placed at the bottom of the concentrator placed on a magnetic stirrer attached to a hand pumpto generate a vortex in the suspension within the concentrator. This magnetic stirring maximisesthe amount of particulates held in suspension throughout the filtration process, and should prevent oocysts from strongly attaching themselves to the membrane.After the liquid has reached a stable rotational velocity, the sample is drained away through the membrane using a vacuum below 40KPa. The membrane is then removedand placed inside a polythene bag containing 5 to $10 \mathrm{~mL}$ of PBST.Once the bag is sealed,the surface of the membrane is rubbed between thumb and forefinger for $70 \pm 10$ seconds until the membrane appears to be clean. Finally, the eluent liquid is removed using a plastic Pasteur pipette and added to a $50 \mathrm{~mL}$ centrifuge tube with the concentrate fraction obtained from the rinsed stirrer bar. The addition of 5-10mL of PBST and rubbing is repeated a second time and the volume in the centrifuge tube made up to $50 \mathrm{~mL}$. The $50 \mathrm{~mL}$ was then passed onto centrifugation, immunomagnetic separation and microscopy for detection and 
enumeration of oocysts.Two elution stages can be distinguished from the above procedure: one from the sponge filter where 1.2L of PBST is used for further sample concentration, the other from the membrane whereby $50 \mathrm{~mL}$ of PBST is employed.

Both stages were studied in this article. In the case of the sponge filters, 1000 litres of uncontaminated water were spiked with 100 oocysts and filtered through the sponge filter over 24 hours. Recovery rates were then measured by carrying out the rest of the traditional process. In the case of the membranes, 100 oocysts in a $1 \mathrm{~mL}$ of water were passed directly through the membrane andrecovery rates were determined by undertaking the rest of the standard procedure.

\subsection{Elution with megasonic sonication}

Atransducer from the Company Sonosys with a frequency of $2 \mathrm{MHz}$ and an output power of 1200 Watts was employed to investigate the elution with megasonic energy assisted agitation(Sonosys. 2015). The encapsulated transducer made of stainless steel was positioned at the bottom side of an existing tankas shown in Figure 1. The sponge filters were added to a large plastic bag with up to $1.2 \mathrm{~L}$ of PBST whereas the membranes were added to the bag utilised in the traditional approach with up to $50 \mathrm{~mL}$ volumes of PBST.

\subsection{Assessment of oocysts viability}

An excystation assay was performed accordingly to protocol. Briefly a sample of 1 million oocysts in $40 \mu \mathrm{L}$ of Hanks Buffered Salt Solution (HBSS) were added to $50 \mu \mathrm{L}$ of trypsin at $\mathrm{pH}=3$ and incubated in a water bath for $60 \mathrm{mins}$ at $37^{\circ} \mathrm{C}$ followed by re-suspension in $90 \mu \mathrm{L}$ HBSS using $10 \mu \mathrm{L}$ sodium bicarbonate and $10 \mu \mathrm{L}$ sodium deoxycholate at $\sim \mathrm{pH}=8$ for $40 \mathrm{mins}$ 145 at the same temperature. An aliquot of the excysted solution was placed on a microscope 
slide and counted under differential interference contrast microscopy for a minimum of 250 counts per sample(Blewett 1989a and Blewett1989b). Three replicates of both the control and the solution treated with megasonic energy were counted. The latter solution was exposed to megasonic agitation for 120 minutes a week before the excystation assay took place. All sampleswere stored in the fridge during that time.

\subsection{Reagents and equipment}

Spiked samplesofCryptosporidium parvum oocysts counted on the flow cytometer (BD Influx ${ }^{\mathrm{TM}}$ cell sorter) were generously provided by Scottish Water. The oocystswere purchased from the company Creative Science, spin out company from the Moredun Institute, which produced and isolated these oocysts. Oocysts used for the experiments were prepared about two months before tests took place and were stored in the fridge. The filters utilised are FiltaMax Filter Modules from IDEXX (Idexx. 2015)and all other reagents were from Cellabs Pty Ltd.

Figure 1: Experimental set-up for the elution using megasonic sonication. The sponge filters, seen at the top of the figure have a doughnut shape when fully expanded and are enclosed in a plastic bag. The membrane is seen in a smaller bag on the bottom left of the figure. The megasonic transducer, seen as a black square, is placed at the bottom of the bath filled with water. 


\section{Results}

\subsection{Influence of sonication time duringelution on oocysts recovery rate}

\subsubsection{Sonication of membranes}

Figure 2: Recovery rates using elution with megasonic energy assisted agitation.

$50 \mathrm{~mL}$ elution volumes, 100 oocysts spiked into $1 \mathrm{~mL}$ were passed through the membrane using the traditional set-up. One experiment was carried out at 2, 4, 6, 8, 10 and 120 minutes and two experiments were carried out at 20, 30, 40, 50 and 60 minutes. 


\subsubsection{Sonication of the IDEXX filters and membranes}

Figure 3 showscomparison between controlled tests carried out without megasonic agitation as in the normal procedure and tests with megasonic agitation. In the case of the filters,the control samples underwent traditional process using $1200 \mathrm{~mL}$ of PBST in the first stage and $50 \mathrm{~mL}$ in the second stage followedby centrifugation, IMS and microscope detection of the stained oocysts. For the megasonic samples, the sponge filters were eluted inside the megasonic bath using $1200 \mathrm{~mL}$ of PBST for 20 minutes and then traditional membrane elution was used for the $2^{\text {nd }}$ stage. The control sample for the membranes underwent traditional process and involving manual rubbing of the membrane. The megasonic sample was eluted into $50 \mathrm{~mL}$ of PBST for 20minutes.

The data in Figure 3 were analysed statistically using a one-way Analysis of Variance (ANOVA) test to examine whether there was a significant effect of the use of megasonic energy in the recovery rate of the oocysts during the elution of filters and membranes. The analysis was carried out using Microsoft Excel program for Windows 8 package. The F-ratios was $\mathrm{F}=0.606$ for the filters and $\mathrm{F}=0.01$ for the membranes, both ratios being less than the critical F-ratio, $\mathrm{F}_{\text {crit }}(0.05,1,4)=7.709$, indicating thereby that the analysis fails to reject the null hypothesis of major difference in the achieved recovery rates between the control and the elution carried out using megasonic assisted agitation. There is therefore no significant difference in terms of enhanced recovery rate.

Figure 3: Comparison between controlled tests without megasonic agitation as in the normal procedure and tests with megasonicagitationfor both filters and membranes. Results obtained for an average of 3 replicates, spiked with 100 oocysts. 


\subsection{Performance of megasonic elution at different volumes}

Filters were placed inside a plastic bag with different volumes of PBST to study the performance of megasonic elution. Table 1 shows that the recovery rate increases with the volume of PBST at the $1^{\text {st }}$ stage of the elution.

Table 1: Recovery rate of filter sonicatedfor 40 minutes using different volumes of PBST. Filters were spiked with 100 oocysts.)

Membranes were also placed inside a plastic bag with different volumes of PBST to study the performance of megasonic elution at different volumes. Table 2 shows that the recovery rate falls slightly, from $66 \%$ to $53 \%$, when the volume of PBST in the $2^{\text {nd }}$ stage elution is decreased from $50 \mathrm{~mL}$ to $15 \mathrm{~mL}$.

Table 2: Recovery rate of membrane sonicated for 20 minutes using different volumes of PBST.

Membranes were spiked with 100 oocysts.

\section{A full procedure for megasonic elution}

The previous results investigated the impact of megasonic elution for each of the different filtration stages, demonstrating that 20minutes of megasonic elution is sufficient to match recovery rates achieved by the existing protocol. Thus the main advantage of utilising megasonic elution is in replacing the existing elution method with an easy to use, automatable approach. Additionally, the use of megasonic elution reduces operator variability and should increase the reproducibility of the results in terms of recovery rates of the pathogens. A key finding of this work is that the volume of elution solution can be 
reducedsuch that megasonic elution would allowthe centrifugation stage of the traditional process to be skipped as $10 \mathrm{~mL}$ would be sufficient to achieve the same recovery rate as in the traditional method for the membrane alone as described in Figure 4. This is confirmed by a single factor Analysis of Variance which shows no significant difference between the recovery rate of oocysts with megasonic energy for both stages and the control tests as the Fratio is $\mathrm{F}=2.41$ which is less than $\mathrm{F}_{\text {crit }}(0.05,1.4)=7.709$.

244 Figure 5 presents a timeline of the existing and proposed elution methods using megasonic agitation. In addition to removing a stage that is challenging to automate (centrifugation), the sonicated elutionfor one sample saves approximately 15 minutesand about $600 \mathrm{~mL}$ of PBST. Although centrifugation could simultaneously process 20 samples,time saving scales with the number of samples as the membrane rubbing cannot be scaled up without increasing the number of operators. However, all membranes could be processed in one step for the megasonic elution approach. Thus the time savings for 20 samples would become 1 hour 50 minutes.

Figure 4: Recovery rates of controlled tests without megasonic and full megasonic elution using $600 \mathrm{ml}$ in the first stage and only $10 \mathrm{ml}$ in the $2^{\text {nd }}$ stage elution and detection process without centrifugation.

Figure 5: Schematic timeline describing the savings in terms of process time and volume of PBST of the megasonic elution compared to the traditional elution method.

\section{Impact of megasonic sonication on oocyst viability}

259 Destruction of oocysts during the elution procedure is obviously undesirable; additionally inactivation of oocysts would preventany subsequent determination of infectivity. Therefore 
experiments were carried out to check the impact of megasonic energy on oocysts. However, an excystation assay is considered a more reliable means of assessing oocyst viability and this test was subsequently performed. The results are shown in Table 3, clearly showing no difference in excystation rate (percentage excystation) or in the sporozoite/shell ratio between the control sample and the sample exposed to elution by megasonic sonication. The excystation assay was performed a week after the megasonic exposure to confirm that the megasonic agitation did not have influence oocyst viability via a slow acting mechanism, which might not have been observed had the excystation assay been undertaken immediately after megasonic exposure.

Table 3: Results of the excystation assay

\section{Conclusions}

This article reports the first investigation of a novel physical approach for the elution of filters and membranes used in waterborne pathogen monitoring. It is demonstrated that the sonication of filters and membranesusing a megasonic transducer preserves the viability of oocysts and achieves recovery rates similar to the established sampling procedure. The key advantages of this novel method lie in (1) the decrease of the volume of the reagents required, (2) the reduction of the manual intervention needed, (3) the reduction of time and resourcesand (4) the potential for automation. In order to fully document the economic impact of this new elution method, arobust cost of ownership (COO) assessment should be undertaken.Whereas the performance of this method has been demonstrated with Cryptosporidium, an extension of theuse of sonication for elution to other pathogens is obviously desirable. The next steps are to work towards an automated filtration/elution 
system based on megasonic sonication assisted elution. As centrifugation would no longer be required, this set-up could then easily be integrated with automated IMS and detection protocols for a fully automated solution to waterborne pathogen monitoring.

\section{Acknowledgements}

The authors express their thanks to Susan Lee, James Green (Scottish Water) as well as Ben Horton (Moredun Scientific Ltd) for their time, assistance and help, which contributed to the development of this article. Their contribution to the assessment of the recovery rates and provision of oocysts are also gratefully acknowledged. This study was made possible through the financial support of the European Union through the project entitled "Aquavalens" (grant number 311846, theme KBBE-2012-2.5-01). Dr Bridle would like to acknowledge the funding the Royal Academy of Engineering and Engineering \& Physical Science Research Council (EPSRC) for her research Fellowship.

\section{References}

Al-Sabi, M.N.S., Gad, J., Klinting, M., Mogensen, C., Kurtzhals, J., Enemark, H.L., 2011. Effects of ultrasound on the survival and characteristics of Cryptosporidium oocysts and Giardia cysts.Proceedings 23rd WAAVP 2011. ed. / G. Mauricio Bulman. Ciudad Autónoma de Buenos Aires: AAPAVET.

Ashokkumar, M., Vu, T., Grieser, F., Weerawardena, A., Anderson, N., Pilkington, N., Dixon, D.R., 2003. Ultrasonic treatment of Cryptosporidium oocysts.Journal of Water Science and Technology 47 (3). 173-177.

Blewett, D.A. 1989a. Quantitative techniques in cryptosporidium research. In K.W. Angus and D.A. Blewett (Ed.)Cryptosporidiosis: Proceedings of the First International Workshop.Moredun Research Institute, Edinburgh United Kingdom. 85-95. 
308

Blewett, D.A. 1989b. Disinfection and oocysts. In: K.W. Angus, D.A. Blewett (Eds.),

Proceedings of the First International Workshop. Moredun Research Institute, Edinburgh

United Kingdom. 107-115.

Bridle, H., Kersaudy-Kerhoas, M., Miller, B., Gavriilidou, D., Katzer, F., Innes, E.A., Desmulliez, M.P., 2012. Detection of Cryptosporidiumin miniaturised fluidic devices. Water Research 46 (6), 1641-1661.

Bridle, H., 2013. Waterborne Pathogens, detection methods and applications. Book: Academic Press, Elsevier, ISBN: $\quad$ 978-0-444-59543-0.

Bridle, H., Miller, B., Desmulliez, M.P., 2014. Application of microfluidics in waterborne pathogen monitoring: a review. Water Research 15 (55), 256-271.

Brugha, R., Vipond, I.B., Evans, M.R., Sandifer, Q.D., Roberts, R.J., Salmon, R.L., Caul, E.O., Mukerjee A.K.,1999. A community outbreak of food-borne small roundstructured virus gastroenteritis caused by a contaminated water supply. Epidemiology and Infection, 122, $145-154$

Busnaina, A.A., Kashkoush,I.I., Gale,G.W., 1995. An experimental-study of megasonic cleaning of siliconwafers.Journal of The Electrochemical Society 142 (8), 2812-2817.

Chitra, S., Paramasivan, K., Sinha, P., Lal, K., 2004. Ultrasonic treatment of liquid waste containing EDTA” Journal of cleaner production 12(4), 429-435.

Francy, D.S., Stelzer, E.A., Brady, A.M., Huitger, C., Bushon, R.N., Ip, H.S.,Ware, M.W., Villegas, E.N., Gallardo, V., Lindquist, H.D.,2013. Comparison of filters for concentrating microbial indicators and pathogens in lake water samples.Applied and Environment Microbiology79(4), 1342-1352. 
Helbig, S., Urban, S., Klein, E., Singh, S., 2008. Impact of megasonic process conditions on PRE and sub-resolutionassist feature damage. Proceedings of SPIE (7122) Photomask Technology 2008, 71221001-71221010.

Hrudey, S.E., Payment, P., Huck, P.M., Gillham, R.W. Hrudey, E.J. 2003. A fatal waterborne disease epidemic in Walkerton,Ontario: comparison with other waterborne outbreaks inthe developed world. Water Science and Technology, Vol 47, No 3, 7-14.

Idexx. 2015. Filta-Max Filter Modules from IDEXX company, http://www.idexx.co.uk/resource-library/water/filta-max-procedure.pdf. (Last accessed 09/05/2015)

Kaufmann, J.,Desmulliez, M.P.Y., Price, D., Hughes, M., Strussevich, N., Bailey, C., 2008.Influence of megasonic agitation on the electrodeposition of high aspect ratio blind vias. Electronics System-Integration Technology Conference, ESTC 1-4 September 2008 IEEE Xplore Digital Library.

Leskinen, S.D., Brownell M., Lim D.V., Harwood, V.J.,2010. Hollow-fiber ultrafiltration and PCR detection of human-associated genetic markers from various types of surface water in Florida.Applied and Environment Microbiology76(12): 4116-4117.

Mendez, J., Audicana, A., Isern, A., Llaneza, J., Moreno, B., Tarancon, M.L., Jofre, J., Lucena, F., 2004. Standardised evaluation of the performance of a simple membrane filtration-elution method to concentrate bacteriophages from drinking water. Journal of Virological Methods 117 (1), 19-25.

Method 1623.1, 2012. Method 1623.1: Cryptosporidium and Giardia in Water by Filtration/IMS/FA.Environmental Protection Agency, United States 
Mull, B., Hill, V.R., 2009. Recovery and detection of Escherichia coli O157:H7 in surface water, using ultrafiltration and real-time PCR.Applied and Environment Microbiology75 (11), 3593-3597.

Polaczyk, A.L., Narayanan, J., Cromeans, T.L., Hahn, D. Roberts, J.M., Amburgey, J.E. Hill, V.R., 2008. Ultrafiltration-based techniques for rapid and simultaneous concentration of multiple microbe classes from $100 \mathrm{~L}$ tap water samples. Journal of Microbiological Methods 73(2), 92-99.

Ruban, S.W., Sharada, R., Banday, T.G., 2011. Physical methods of separation and concentration of microbes in food: an aid for rapid detection. Journal of Food Technology 9 (3), 106-111.

Smith, C., Hill, V.R., 2009. Dead-end hollow fiber ultrafiltration for recovery of diverse microbes from water.Applied and Environment Microbiology75 (16), 5284-5289.

Söderström, A., Osterberg, P., Lindqvist, A., Jönsson, B., Lindberg, A., Blide Ulander, S., Welinder-Olsson, C., Löfdahl, S., Kaijser, B., De Jong, B., Kühlmann-Berenzon, S., Boqvist, S., Eriksson, E., Szanto, E., Andersson, S., Allestam, G., Hedenström, I., Ledet Muller, L., Andersson, Y.,2008. A large Escherichia coli O157 outbreak in Sweden associated with locally produced lettuce. Foodborne Pathogens and Disease 5(3), 339-349.

Sonosys. 2015.The megasonic systemis from the Sonosys Company

http://www.sonosys.de/products/ submersible-transducer. (Last accessed 09/05/2015).

UK Environment Agency, 2010. Methods for theisolation, identification and enumeration of Cryptosporidium oocystsand Giardia cysts. The United Kingdom Environment Agency Blue Book publications (Microbiology of Drinking Water, Part 14). 
374 World Health Organisation, 2011. Guidelines for drinking-water quality. Fourth editionISBN:

1

3759789241548151 .

4

5

6376

7

8

9

10

11

12

13

14

15

16

17

18

19

20

21

22

23

24

25

26

27

28

29

30

31

32

33

34

35

36

37

38

39

40

41

42

43

44

45

46

47

48

49

50

51

52

53

54

55

56

57

58

59

60

61

62

63

64

65 
Table 1: Recovery rate of filter sonicatedfor 40 minutes using different volumes of PBST. Filters were spiked with 100 oocysts. $(n=3$ trials $)$

\begin{tabular}{ccc}
\hline Volume of PBST $(\mathbf{m L})$ & Mean recovery rate $(\boldsymbol{\%})$ & Standard deviation (SD) \\
\hline $\mathbf{5 0}$ & 4.66 & 2.05 \\
$\mathbf{1 0 0}$ & 16.66 & 2.86 \\
$\mathbf{5 0 0}$ & 25 & 1.63 \\
$\mathbf{6 0 0}$ & 31.33 & 1.69 \\
$\mathbf{1 2 0 0}$ & 38.62 & 2.62 \\
\hline
\end{tabular}


Table 2: Recovery rate of membrane sonicated for 20 minutes using different volumes of PBST.

Membranes were spiked with 100 oocysts. $(n=3$ trials)

\begin{tabular}{ccc}
\hline Volume of PBST $(\mathbf{m L})$ & Mean recovery rate $(\boldsymbol{\%})$ & Standard Deviation (SD) \\
\hline $\mathbf{1 5}$ & 50.33 & 2.05 \\
$\mathbf{2 0}$ & 61 & 1.63 \\
$\mathbf{5 0}$ & 65.66 & 1.24 \\
\hline
\end{tabular}


Table 3: Results of the excystation assay

\begin{tabular}{ccc}
\hline Sample & Excystation percentage & Sporozoite/shell ratio \\
\hline Control $(\boldsymbol{n}=1$ trial $)$ & 97 & 2.4 \\
Megasonic $(\boldsymbol{n}=3$ trials $)$ & Mean value $=96$ & Mean value $=2.26$ \\
& $\mathrm{SD}=1.11$ & $\mathrm{SD}=0.36$ \\
\hline
\end{tabular}




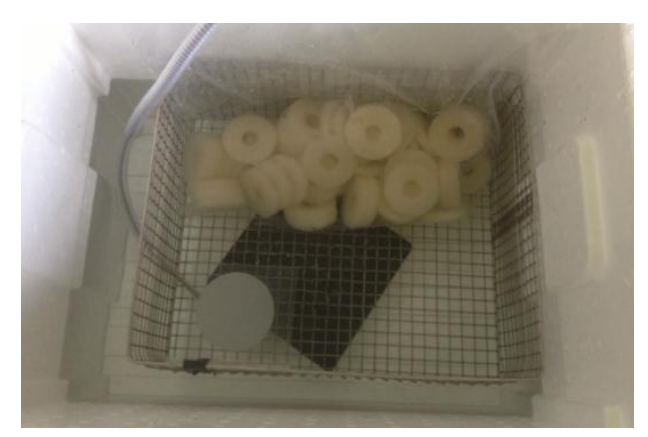

Figure 1: Experimental set-up for the elution using megasonic sonication. The sponge filters, seen at the top of the figure have a doughnut shape when fully expanded and are enclosed in a plastic bag. The membrane is seen in a smaller bag on the bottom left of the figure. The megasonic transducer, seen as a black square, is placed at the bottom of the bath filled with water. 


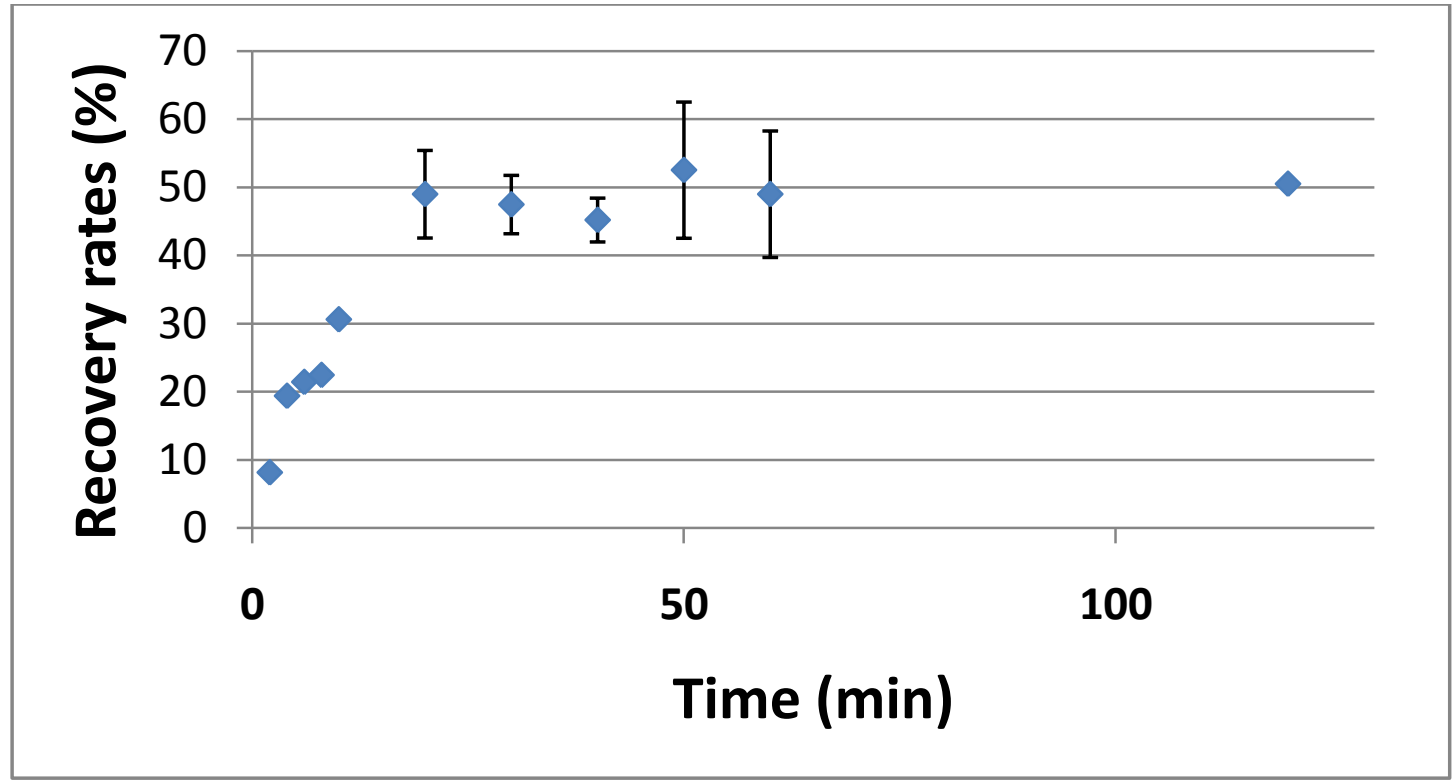

Figure 2: Recovery rates using elution with megasonic energy assisted agitation. 50mL elution volumes, 100 oocysts spiked into $1 \mathrm{~mL}$ were passed through the membrane using the traditional set-up. One experiment $(n=1)$ was carried out at 2, 4, 6, 8, 10 and 120 minutes and two experiments $(n=2)$ were carried out at 20, 30, 40, 50 and 60 minutes. 


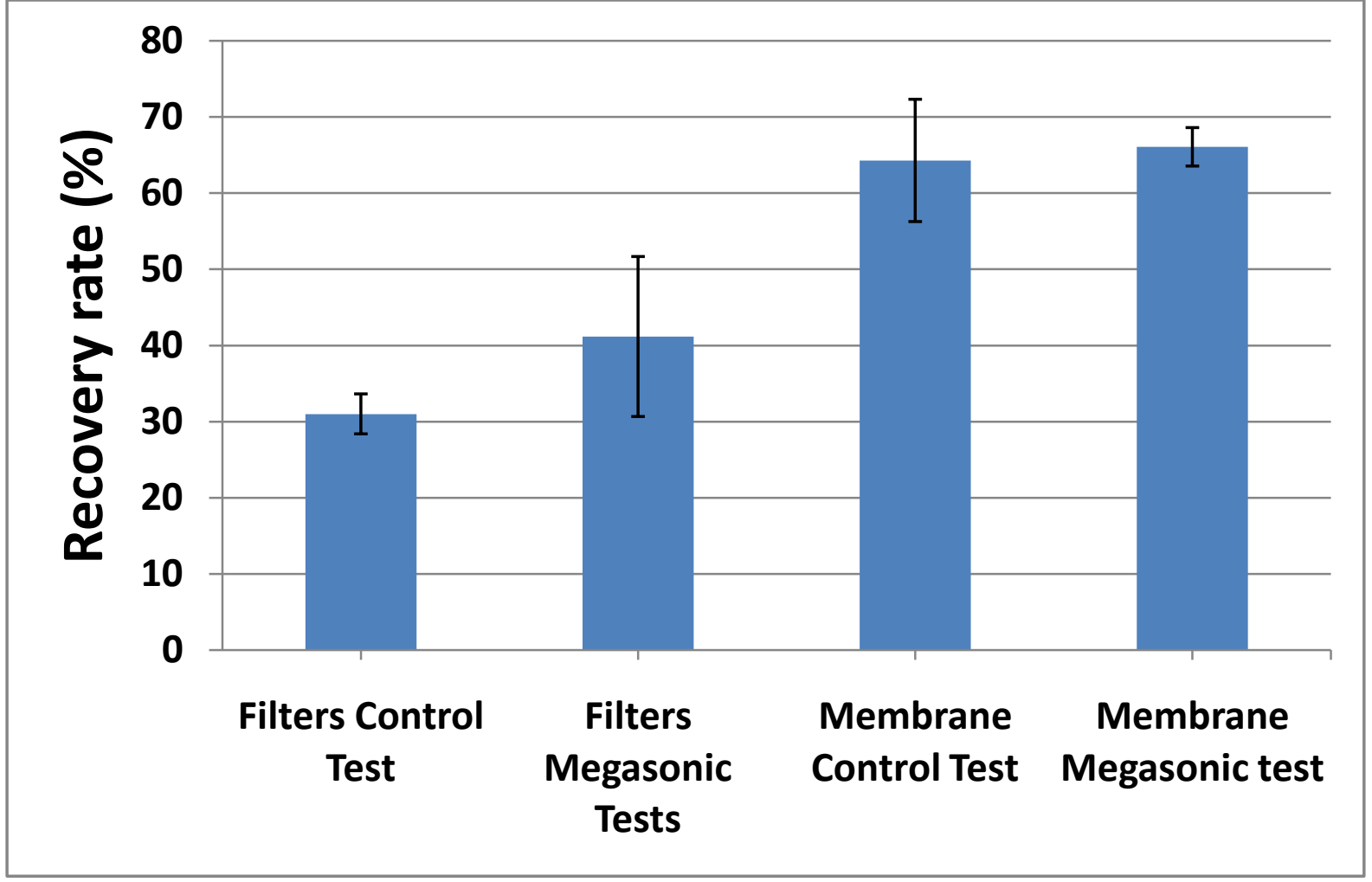

Figure 3: Comparison between controlled tests without megasonic agitation as in the normal procedure and tests with megasonicagitationfor both filters and membranes.

Results obtained for an average of 3 replicates, spiked with 100 oocysts. 


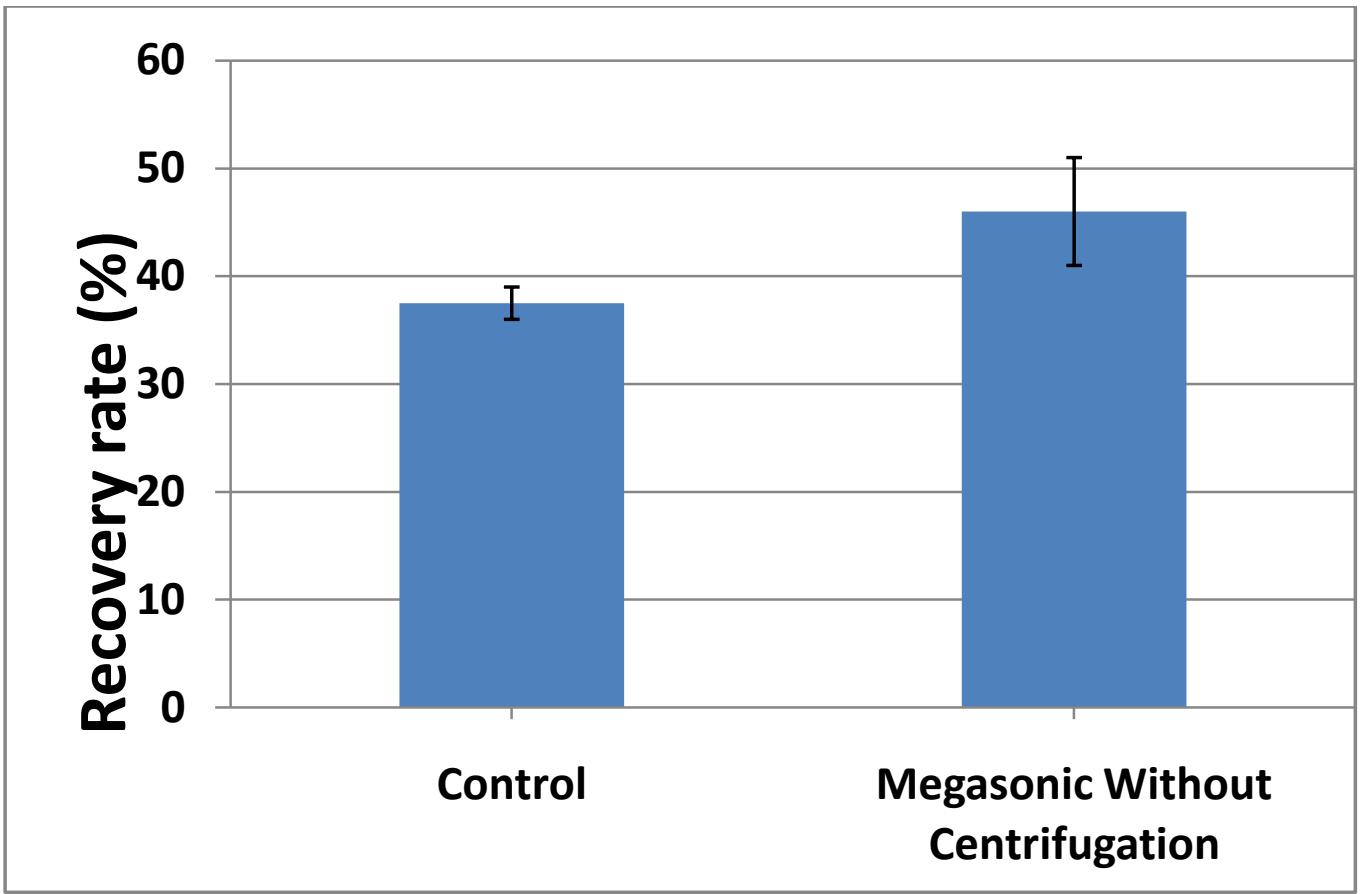

Figure 4: Recovery rates of controlled tests without megasonic and full megasonic elution using $600 \mathrm{ml}$ in the first stage and only $10 \mathrm{ml}$ in the $2^{\text {nd }}$ stage elution and detection process without centrifugation. 


\section{Time Existing method Megasonic elution}

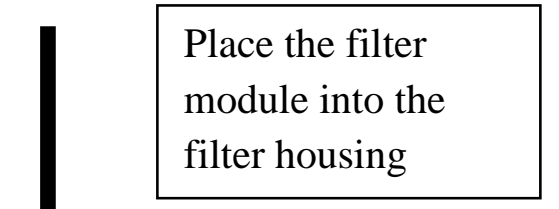

Wash the sample with PBST

$1200 \mathrm{ml}$

20-30 minutes

Concentrate the sample througha membrane

Rub the membrane and put the sample from the bag to a $50 \mathrm{ml}$ centrifuge tube. Add 5-10 ml PBST

2 minutes

\begin{tabular}{l} 
Centrifugation \\
$50 \mathrm{ml}$ Tube \\
$\mathbf{3 0}$ minutes \\
\hline
\end{tabular}

IMS separation

Max: 10ml volume

To detection
Unscrew the filter, place the sponges inside the plastic bag and put the bag into the megasonic bath

$600 \mathrm{ml}$ PBST

\section{Concentrate the} sample througha membrane

\section{Place the} membrane inside the plastic bag and put the bag inside the megasonic bath 5-10 ml PBST

\section{0 minutes}

IMS separation

Max: $10 \mathrm{ml}$ volume

To detection

Figure 5: Schematic timeline describing the savings in terms of process time and volume of PBST of the megasonic elution compared to the traditional elution method. 